\title{
Chronostratigraphy, Site Formation, and Palaeoenvironmental Context of Late Pleistocene and Holocene Occupations at Grassridge Rock Shelter (Eastern Cape, South Africa)
}

Christopher J.H. Ames ${ }^{1,2}$, Luke Gliganic ${ }^{3}$, Carlos E. Cordova ${ }^{4}$, Kelsey Boyd ${ }^{1}$, Brian G. Jones ${ }^{1}$, Lisa Maher ${ }^{5}$, and B.R. Collins $s^{6,7}$

1 - School of Earth, Atmospheric, and Life Sciences, University of Wollongong

2 - Department of Anthropology, University of Victoria

3 - Institute of Geology, University of Innsbruck

4 - Department of Geography, Oklahoma State University

5 - Department of Anthropology, University of California-Berkeley

6 - Department of Anthropology, University of Manitoba

7 - Department of Archaeology, University of Cape Town

\section{Stratigraphic and Sedimentological Field Observations and Laboratory Data}

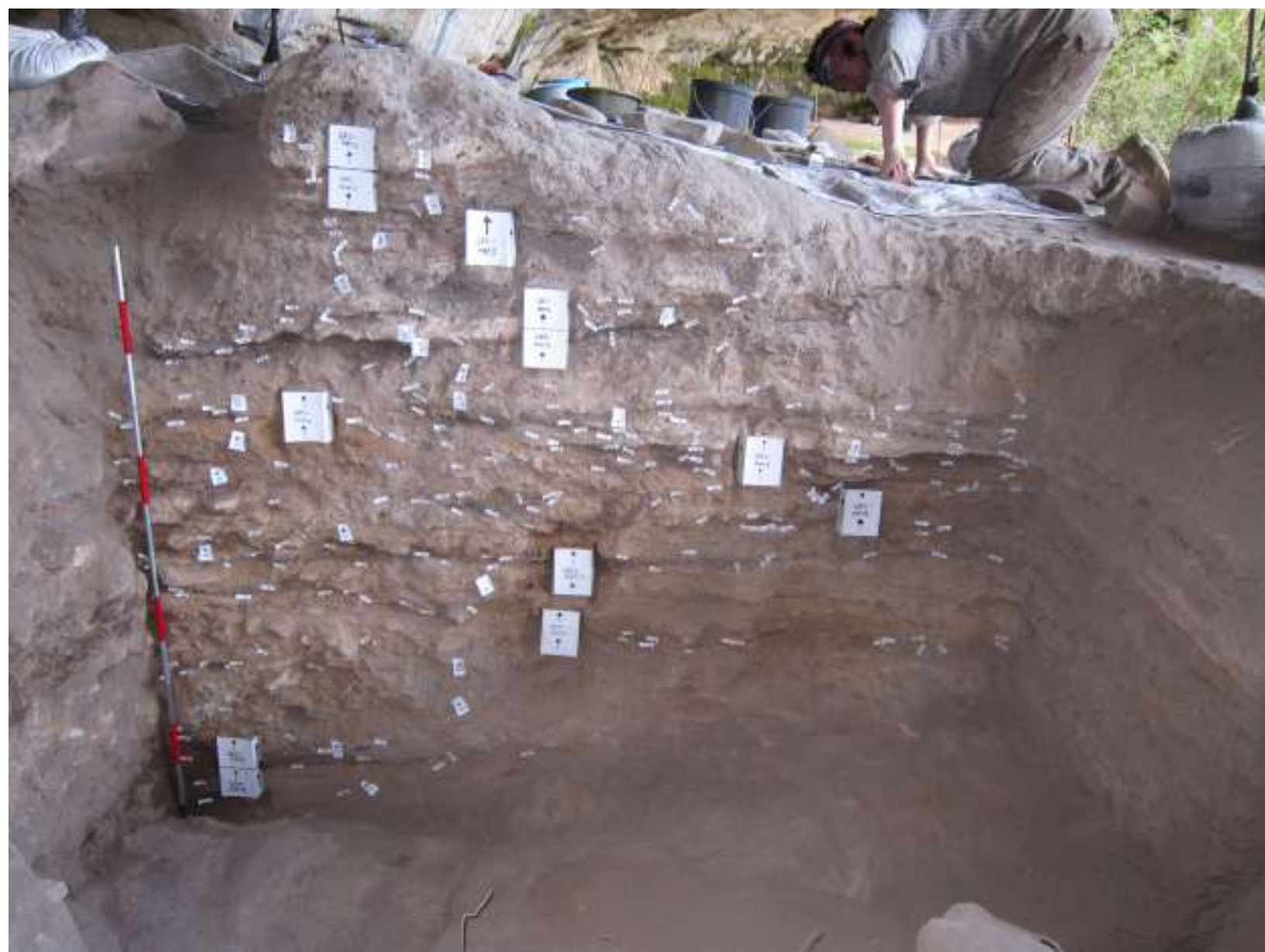

Figure S2.1 B-C 2/3 Stratigraphic Profile with each stratigraphic unit labeled with wall tags and showing the locations of micromorphological samples, although the results of the latter are not discussed in this manuscript (red scale bar segments are $10 \mathrm{~cm}$ in length). 
Table S2.1 Field descriptions of stratigraphic units identified in the B-C 2/3 stratigraphic sequence (SU = stratigraphic unit). Refer to Figure 5 and 6 in the manuscript for additional information.

\begin{tabular}{|c|c|c|c|c|c|c|c|}
\hline SU & $\begin{array}{l}\text { Colour } \\
\text { (Munsell) }\end{array}$ & $\begin{array}{l}\text { Thickness } \\
\text { (cm) }\end{array}$ & Texture & Consistence & Structure & $\begin{array}{l}\text { Lower } \\
\text { Boundary }\end{array}$ & Comments \\
\hline 1 & 10YR $4 / 2$ & $4-11$ & Clayey silt & $\begin{array}{l}\text { Moderately } \\
\text { hard }\end{array}$ & Massive & Clear & $10 \%$ scattered charcoal (5-10 $\mathrm{mm}$ pieces) \\
\hline 2 & 10YR $6 / 2$ & 3 & Sandy silt & Slightly hard & Massive & Abrupt & $5 \%$ scattered charcoal (5-10 mm pieces) \\
\hline 3 & $10 Y R 3 / 3$ to $4 / 3$ & $1-2$ & $\begin{array}{l}\text { Slightly } \\
\text { sandy loam }\end{array}$ & Single grain & Loose & $\begin{array}{l}\text { Clear to } \\
\text { Diffuse }\end{array}$ & At contact between units 2 and 4 \\
\hline 4 & 10YR $6 / 2$ & $1-2$ & $\begin{array}{l}\text { Slightly } \\
\text { sandy silt }\end{array}$ & Slightly hard & Massive & Clear & Thin layer within unit 5 \\
\hline 5 & 10 YR $5 / 3$ & $6-8$ & Sandy silt & Soft & Massive & Clear & $15 \%$ scattered charcoal ( $\sim 5 \mathrm{~mm}$ pieces) \\
\hline 6 & 10YR 5/4 & 1 & $\begin{array}{l}\text { Not } \\
\text { recorded }\end{array}$ & Slightly hard & Massive & Clear & $\begin{array}{l}\text { Only } 20 \mathrm{~cm} \text { width with } 5 \% \text { scattered charcoal } \\
\text { (<5 mm pieces) }\end{array}$ \\
\hline 7 & 10 YR $3 / 3$ to $4 / 3$ & $1-2$ & $\begin{array}{l}\text { Sandy silt } \\
\text { loam }\end{array}$ & $\begin{array}{l}\text { Slightly hard } \\
\text { to soft }\end{array}$ & $\begin{array}{l}\text { Massive to } \\
\text { Loose }\end{array}$ & $\begin{array}{l}\text { Clear to } \\
\text { abrupt }\end{array}$ & At contact between units 5 and 8 \\
\hline 8 & 10YR $5 / 2$ & $2-4$ & $\begin{array}{l}\text { Sandy silt } \\
\text { loam }\end{array}$ & Soft & Massive & Clear & $5 \%$ scattered charcoal (5-10 mm pieces) \\
\hline 9 & $7.5 Y R 4 / 4$ & $1-2$ & $\begin{array}{l}\text { Sandy } \\
\text { silt/silty } \\
\text { sand }\end{array}$ & Soft & Loose & Clear & $\begin{array}{l}\text { At contact between units and } 10 \text {. Very similar to } \\
\text { units } 3 \text { and } 7\end{array}$ \\
\hline 10 & $10 Y R 5 / 3$ to $6 / 3$ & $8-10$ & $\begin{array}{l}\text { Sandy } \\
\text { silt/silty } \\
\text { sand }\end{array}$ & $\begin{array}{l}\text { Soft to } \\
\text { slightly hard }\end{array}$ & Massive & Clear & $\begin{array}{l}\text { 10-15\% scattered charcoal (2-10 } \mathrm{mm} \text { pieces), } \\
\text { visible bone and stone artefacts, very few } 1 \mathrm{~mm} \\
\text { diameter roots, and evidence of one small } \\
\text { burrow }\end{array}$ \\
\hline 11 & $10 Y R \quad 4 / 3$ to $4 / 4$ & $1-2$ & Sandy silt & Soft & Loose & Abrupt & $\begin{array}{l}\text { Bone and stone artefacts visible, and very few } 1 \\
\mathrm{~mm} \text { diameter roots }\end{array}$ \\
\hline 12 & 10YR $7 / 3$ & $2-5$ & $\begin{array}{l}\text { Slightly } \\
\text { sandy silt }\end{array}$ & $\begin{array}{l}\text { Moderately } \\
\text { hard }\end{array}$ & Massive & Abrupt & $\begin{array}{l}\text { Homogeneous layer similar to unit } 2 \text { but } \\
\text { without the charcoal }\end{array}$ \\
\hline
\end{tabular}




\begin{tabular}{|c|c|c|c|c|c|c|c|}
\hline & & & sandy silt & & $\begin{array}{l}\text { laminar to } \\
\text { massive }\end{array}$ & & 2 and 12 \\
\hline 14 & 10YR $5 / 3$ & $2-5$ & Silty sand & Soft & Massive & Clear & $\begin{array}{l}\text { Similar to unit } 10 \text { with very few } 1 \mathrm{~mm} \text { diameter } \\
\text { roots }\end{array}$ \\
\hline 15 & $7.5 Y R 3 / 2$ to $2 / 5 / 2$ & $1-4$ & Silty loam & Very soft & Loose & Clear & $\begin{array}{l}\text { Common } 1-2 \mathrm{~mm} \text { diameter roots with many } \\
\text { visible artefacts }\end{array}$ \\
\hline 16 & 10YR $4 / 4$ & $6-10$ & $\begin{array}{l}\text { Slightly } \\
\text { sandy silt }\end{array}$ & Slightly hard & $\begin{array}{l}\text { Weak sub- } \\
\text { rounded to } \\
\text { massive }\end{array}$ & $\begin{array}{c}\text { Gradual with } \\
\text { unit } 20 / \\
\text { diffuse with } \\
\text { unit } 17\end{array}$ & $\begin{array}{l}\text { Some visible artefacts and common } 1-2 \mathrm{~mm} \\
\text { diameter roots }\end{array}$ \\
\hline 17 & 10YR 5/4 & $2-4$ & Silt & Very soft & $\begin{array}{l}\text { Massive to } \\
\text { loose }\end{array}$ & Diffuse & \\
\hline 18 & 10YR $4 / 3$ to $4 / 4$ & $2-5$ & Sandy silt & $\begin{array}{l}\text { Moderately } \\
\text { hard }\end{array}$ & Massive & $\begin{array}{c}\text { Clear to } \\
\text { abrupt }\end{array}$ & Very few $1-2 \mathrm{~mm}$ diameter roots \\
\hline 19 & 10YR $4 / 4$ & $1-3$ & Silty sand & Soft & Loose & Clear & \\
\hline 20 & 10YR $5 / 3$ & $1-2$ & Sandy silt & Soft & Massive & $\begin{array}{l}\text { Clear to } \\
\text { diffuse }\end{array}$ & Similar to units 5 and 10 \\
\hline 21 & $10 Y R 4 / 3$ to $4 / 4$ & $2-5$ & Sandy silt & $\begin{array}{l}\text { Moderately } \\
\text { hard }\end{array}$ & Massive & $\begin{array}{l}\text { Clear to } \\
\text { abrupt }\end{array}$ & \\
\hline 22 & $7.5 Y R 2.5 / 2$ & $1-3$ & Sandy loam & Soft & Loose & $\begin{array}{l}\text { Clear to } \\
\text { Abrupt }\end{array}$ & $\begin{array}{l}\text { Few } 1-2 \mathrm{~mm} \text { roots; small lens-shaped unit with } \\
5 \% \text { scattered charcoal (1-3 } \mathrm{mm} \text { pieces) }\end{array}$ \\
\hline 23 & 10YR $7 / 2$ to $6 / 3$ & $1-5$ & $\begin{array}{l}\text { Slightly } \\
\text { sandy silt }\end{array}$ & Slightly hard & $\begin{array}{c}\text { Massive } \\
\text { and weakly } \\
\text { laminar in } \\
\text { areas }\end{array}$ & $\begin{array}{l}\text { Abrupt to } \\
\text { clear }\end{array}$ & Similar to units 10 and 12 \\
\hline 24 & $7.5 Y R 3 / 2$ & $1-2$ & Silty sand & $\begin{array}{c}\text { Very } \\
\text { soft/none }\end{array}$ & Loose & Clear & Lens-shaped feature like unit 22 \\
\hline 25 & 10YR 5/6 & $2-4$ & Sand & Soft & Loose & Abrupt & $\begin{array}{l}\text { Inter-digitates with units } 23 \text { and } 24 \text {, is slightly } \\
\text { darker where it underlies unit } 23 \text {, and has few } \\
1-3 \mathrm{~mm} \text { diameter toots }\end{array}$ \\
\hline 26 & 10YR $3 / 2$ & $1-2$ & Silty sand & Soft & Loose & Clear & Few $1 \mathrm{~mm}$ diameter roots \\
\hline
\end{tabular}




1-3 Rock Very hard $\begin{gathered}\text { Abrupt to } \\ \text { clear }\end{gathered}$ Platy/laminar Carbonate crust

30 10YR $3 / 3$

$1-3$

Silty sand

Very soft$$
31
$$

10YR $4 / 4$ to $3 / 6$

2-8

49

7.5YR $3 / 2$

$1-4$

2-15

Sandy silt

Soft

33

34

36

37

38

39

50
10YR 3/6

10YR $3 / 2$

10YR 5/4

6-17

$5-12$

10YR $4 / 2$

10YR $4 / 4$

2

2

10YR 4/4

3-7

10YR $3 / 3$

1-2

10YR 4/4
Soft

$$
\begin{gathered}
\text { Very fine } \\
\text { sand }
\end{gathered}
$$

Silty sand

Slightly to
moderately
hard

hard

Soft

Soft

Soft

Soft

Silty sand

Silty loam

Loose

Sand
Massive to single

grain

Diffuse

$5 \%$ scattered charcoal (2-5 $\mathrm{mm}$ pieces)

Clear to diffuse

Clear

Loose

Massive

Clear to diffuse

Loose

Massive

Massive

(ose

Loose

Loose

Massive

Single

grain

Soft Massive
Clear to

abrupt

Clear

Diffuse to clear

Clear

Clear to abrupt

Clear to abrupt

Clear

Clear to diffuse
Lens-shaped feature with $25 \%$ charcoal
$15 \%$ scattered charcoal in lower half $(1-10 \mathrm{~mm}$ pieces), and visible artefacts

Lens-shaped feature with 35\% charcoal (5-10 $\mathrm{mm}$ pieces and larger), traces of ash at upper boundary, and few $1 \mathrm{~mm}$ diameter roots

Similar to unit 31 with $5 \%$ scattered charcoal (1-5 $\mathrm{mm}$ pieces) and very few $1 \mathrm{~mm}$ diameter roots (including pieces $>10 \mathrm{~mm}$ ) and few $1-3 \mathrm{~mm}$ diameter roots

2-5\% scattered charcoal (1-3 mm pieces), visible artefacts, and few $1-3 \mathrm{~mm}$ roots.

Very few 1-3 $\mathrm{mm}$ diameter roots

Thin lens-shaped charcoal-rich layer

Lots of charcoal, including relatively large fragments

Common $1-5 \mathrm{~mm}$ roots and $<2 \%$ scattered charcoal (1-3 $\mathrm{mm}$ pieces)

Thin band of charcoal-rich sediment

Similar to unit 38 with few $1-5 \mathrm{~mm}$ diameter roots and $5 \%$ scattered charcoal $(1-5 \mathrm{~mm}$ pieces) 


\begin{tabular}{|c|c|c|c|c|c|c|c|}
\hline 51 & 10YR $3 / 3$ & $1-3$ & Silty sand & Soft & Loose & $\begin{array}{l}\text { Clear to } \\
\text { diffuse }\end{array}$ & $\begin{array}{l}\text { Common } 1-10 \mathrm{~mm} \text { diameter roots and } 15 \% \\
\text { scattered charcoal ( } 1-5 \mathrm{~mm} \text { pieces) }\end{array}$ \\
\hline 40 & & $3-14$ & $\begin{array}{l}\text { Gravel (3-15 } \\
\mathrm{cm})\end{array}$ & & & Clear & Large rocks with no matrix; possibly roof fall \\
\hline 41 & 10YR $5 / 6$ to $4 / 6$ & $1-4$ & Sand & Loose & $\begin{array}{l}\text { Single } \\
\text { grain to } \\
\text { massive }\end{array}$ & $\begin{array}{l}\text { Clear to } \\
\text { diffuse }\end{array}$ & Thin layer between rock units 40 and 43 \\
\hline 42 & 10YR $3 / 3$ & $1-2$ & $\begin{array}{l}\text { Not } \\
\text { recorded }\end{array}$ & $\begin{array}{l}\text { Not } \\
\text { recorded }\end{array}$ & $\begin{array}{l}\text { Not } \\
\text { recorded }\end{array}$ & Clear & $\begin{array}{l}\text { Thin, charcoal-rich layer under unit } 41 \text {, between } \\
\text { rock units } 40 \text { and } 43\end{array}$ \\
\hline 43 & & $4-12$ & $\begin{array}{l}\text { Gravel and } \\
\text { boulder }\end{array}$ & & & Clear & Greenish, gray soft rock fragments \\
\hline 44 & 10YR $5 / 6$ & $2-30$ & $\begin{array}{l}\text { Slightly silty } \\
\text { sand }\end{array}$ & $\begin{array}{l}\text { Moderately } \\
\text { hard }\end{array}$ & Massive & $\begin{array}{l}\text { Clear to } \\
\text { diffuse }\end{array}$ & $\begin{array}{l}15 \% \text { small to medium gravels overall, but } 25 \% \\
\text { large gravels in lower right }\end{array}$ \\
\hline 45 & 10YR $4 / 4$ & $1-3$ & Sand & Hard & Massive & Diffuse & Artefacts visible \\
\hline 46 & 7.5YR $2.5 / 3$ & $2-9$ & Sandy loam & Slightly hard & Massive & $\begin{array}{l}\text { Cleary to } \\
\text { abrupt }\end{array}$ & $\begin{array}{l}10 \% \text { scattered charcoal ( } 1-3 \mathrm{~mm} \text { pieces) and } \\
\text { black mottles }\end{array}$ \\
\hline 48 & 7.5YR $2.5 / 2$ & $1-2$ & Loam & Soft & Massive & Clear & $\begin{array}{l}\text { Charcoal-rich lens with brown mottles and 5- } \\
10 \% \text { scattered charcoal ( } 1-5 \mathrm{~mm} \text { pieces) }\end{array}$ \\
\hline 47 & 10YR 5/6 & $1--15$ & $\begin{array}{l}\text { Slightly silty } \\
\text { sand }\end{array}$ & Hard & Massive & $\begin{array}{c}\text { Abrupt } \\
\text { (bedrock } \\
\text { below) }\end{array}$ & $\begin{array}{l}\text { Similar to unit } 45 \text { but separate by charcoal-rich } \\
\text { unit } 46\end{array}$ \\
\hline
\end{tabular}


Table S2.2 Sedimentological laboratory data (SU = stratigraphic unit).

\begin{tabular}{|c|c|c|c|c|c|c|c|c|c|c|c|c|c|c|c|}
\hline SU & $\begin{array}{l}\text { Depth } \\
\text { Below } \\
\text { Surface } \\
(\mathrm{cm})^{*} \\
\end{array}$ & $\begin{array}{c}\% \\
\text { Charcoal } \\
\end{array}$ & $\begin{array}{c}\% \\
\text { Artefacts } \\
\end{array}$ & $\mathrm{pH}$ & $\begin{array}{c}\% \\
\text { Coarse } \\
\text { Fraction } \\
(>2 \mathrm{~mm})\end{array}$ & $\begin{array}{c}\% \\
\text { Sand }\end{array}$ & $\begin{array}{c}\% \\
\text { Silt }\end{array}$ & $\begin{array}{l}\% \\
\text { Clay }\end{array}$ & $\begin{array}{c}\% \\
\text { Organic } \\
\text { Carbon } \\
\end{array}$ & $\begin{array}{l}\% \text { Clay } \\
\text { Mineral }\end{array}$ & $\begin{array}{c}\% \\
\text { Quartz } \\
\end{array}$ & $\begin{array}{c}\% \\
\text { Feldspar }\end{array}$ & $\begin{array}{c}\% \\
\text { Calcite } \\
\end{array}$ & $\begin{array}{c}\% \\
\text { Hematite }\end{array}$ & $\begin{array}{c}\% \\
\text { Goethite } \\
\end{array}$ \\
\hline 1 & 6.9 & 15.5 & 64.3 & 7.9 & 10.2 & 48.2 & 40.3 & 11.5 & 4.6 & 8.6 & 31.8 & 20.4 & 36.9 & 1.7 & 0.6 \\
\hline 2 & 11.4 & 0.0 & 78.2 & 8.3 & 10.2 & 65.2 & 26.1 & 8.7 & 3.3 & 8.1 & 36.5 & 21.0 & 30.8 & 2.5 & 0.9 \\
\hline 5 & 19.4 & 3.9 & 70.3 & 8.2 & 8.7 & 45.9 & 41.2 & 12.9 & 5.6 & 9.9 & 23.3 & 15.6 & 42.7 & 1.4 & 0.5 \\
\hline 8 & 26.6 & 3.0 & 37.7 & 8.4 & 20.6 & 49.0 & 40.6 & 10.4 & 4.8 & 6.3 & 37.0 & 35.9 & 17.4 & 0.7 & 2.8 \\
\hline 10 & 33.6 & 3.1 & 87.0 & 8.4 & 19.6 & 46.7 & 39.0 & 14.3 & 4.6 & 8.3 & 35.8 & 33.4 & 18.9 & 1.1 & 1.2 \\
\hline 12 & 40 & 0.0 & 39.4 & 8.5 & 6.8 & 50.6 & 37.2 & 12.3 & 4.5 & 10.8 & 47.8 & 18.8 & 20.4 & 1.8 & 0.3 \\
\hline 14 & 43 & 1.3 & 57.1 & 8.6 & 13.7 & 42.2 & 48.9 & 8.9 & 3.8 & 7.8 & 32.4 & 26.7 & 30.5 & 0.9 & 1.7 \\
\hline 15 & 45.7 & 1.3 & 93.4 & 8.5 & 23.3 & 36.0 & 53.5 & 10.5 & 5.1 & 5.9 & 51.4 & 26.9 & 12.9 & 1.1 & 1.5 \\
\hline 16 & 50.6 & 1.1 & 69.3 & 8.6 & 7.8 & 50.4 & 33.2 & 16.4 & 4.3 & 11.0 & 44.4 & 25.1 & 15.3 & 1.7 & 2.4 \\
\hline 17 & 54 & 0.0 & 36.2 & 8.6 & 4.5 & 16.3 & 67.2 & 16.5 & 5.4 & 14.4 & 45.2 & 21.9 & 12.4 & 1.5 & 1.1 \\
\hline 18 & 57 & 1.1 & 83.6 & 8.6 & 10.8 & 49.2 & 37.1 & 13.7 & 4.2 & 6.6 & 31.1 & 44.1 & 15.9 & 1.3 & 0.8 \\
\hline 23 & 62 & 0.0 & 88.4 & 8.7 & 9.1 & 49.2 & 34.5 & 16.3 & 3.7 & 8.7 & 28.2 & 22.9 & 37.8 & 1.8 & 0.6 \\
\hline 25 & 64 & 0.9 & 63.3 & 8.3 & 11.8 & 49.9 & 37.2 & 12.9 & 3.7 & 17.0 & 45.3 & 24.3 & 8.6 & 3.3 & 1.4 \\
\hline 31 & 67 & 7.8 & 58.5 & 8.3 & 3.7 & 19.9 & 52.7 & 27.5 & 7.4 & 16.5 & 44.1 & 33.2 & 0.7 & 2.8 & 2.9 \\
\hline 32 & 71.3 & 1.0 & 12.9 & 8.4 & 15.7 & 40.5 & 47.9 & 11.6 & 6.1 & 16.2 & 66.8 & 15.2 & 0.5 & 0.7 & 0.5 \\
\hline 34 & 83.5 & 0.1 & 1.1 & 8.4 & 18.2 & 53.8 & 25.5 & 20.7 & 3.5 & 15.0 & 72.2 & 10.7 & 0.0 & 0.9 & 1.2 \\
\hline 35 & 87.2 & 0.1 & 3.9 & 8.4 & 18.9 & 49.7 & 33.6 & 16.7 & 4.2 & 18.2 & 63.8 & 16.2 & 0.0 & 0.0 & 1.8 \\
\hline 38 & 95.9 & 0.3 & 1.3 & 8.7 & 20.8 & 50.1 & 39.7 & 10.2 & 3.5 & 7.1 & 68.2 & 21.8 & 0.0 & 0.1 & 2.8 \\
\hline 50 & 104.2 & 0.1 & 0.1 & 8.8 & 17.9 & 61.2 & 27.0 & 11.9 & 1.9 & 9.0 & 70.8 & 18.5 & 0.0 & 0.1 & 1.4 \\
\hline 44 top & 113.2 & 0.1 & 0.2 & 8.7 & 33.3 & 64.4 & 22.6 & 13.0 & 2.2 & 17.7 & 67.7 & 12.3 & 0.3 & 0.4 & 1.6 \\
\hline 44 top & 121.5 & 0.2 & 1.3 & 8.5 & 18.7 & 30.2 & 49.7 & 20.1 & 3.2 & 12.3 & 79.9 & 6.5 & 0.2 & 1.1 & 0.0 \\
\hline 45 & 131.9 & 0.0 & 18.1 & 8.3 & 9.6 & 66.5 & 24.6 & 9.0 & 2.5 & 14.2 & 64.6 & 17.1 & 0.0 & 0.0 & 4.1 \\
\hline 47 & 141.6 & 0.0 & 0.0 & 8.3 & 4.9 & 52.8 & 29.7 & 17.5 & 2.1 & 11.5 & 77.8 & 9.5 & 0.2 & 0.9 & 0.0 \\
\hline
\end{tabular}

*referenced to the uppermost surface elevation for the B-C $2 / 3$ sequence and adjusted to preserve stratigraphic relationships 